\title{
Python Coding of Geospatial Processing in Web-based Mapping Applications
}

\author{
James A. Kuiper ${ }^{\ddagger *}$, Andrew J. Ayers ${ }^{\ddagger}$, Michael E. Holm ${ }^{\ddagger}$, Michael J. Nowak
}

\begin{abstract}
Python has powerful capabilities for coding elements of Web-based mapping applications. This paper highlights examples of analytical geospatial processing services that we have implemented for several Open Source-based development projects, including the Eastern Interconnection States' Planning Council (EISPC) Energy Zones Mapping Tool (http://eispctools.anl.gov), the Solar Energy Environmental Mapper (http://solarmapper.anl.gov), and the Ecological Risk Calculator (http://bogi.evs.anl.gov/erc/portal). We used common Open Source tools such as GeoServer, PostGIS, GeoExt, and OpenLayers for the basic Web-based portal, then added custom analytical tools to support more advanced functionality. The analytical processes were implemented as Web Processing Services (WPSs) running on PyWPS, a Python implementation of the Open Geospatial Consortium (OGC) WPS. For report tools, areas drawn by the user in the map interface are submitted to a service that utilizes the spatial extensions of PostGIS to generate buffers for use in querying and analyzing the underlying data. Python code then post-processes the results and outputs JavaScript Object Notation (JSON)-formatted data for rendering. We made use of PyWPS's integration with the Geographic Resources Analysis Support System (GRASS) to implement flexible, user-adjustable suitability models for several renewable energy generation technologies. In this paper, we provide details about the processing methods we used within these project examples.
\end{abstract}

Index Terms-GIS, web-based mapping, PyWPS, PostGIS, GRASS, spatial modeling

\section{BACKGROUND AND INTRODUCTION}

Web-based mapping applications are effective in providing simple and accessible interfaces for geospatial information, and often include large spatial databases and advanced analytical capabilities. Perhaps the most familiar is Google Maps [Ggl] which provides access to terabytes of maps, aerial imagery, street address data, and point-to-point routing capabilities. Descriptions are included herein of several Web-based applications that focus on energy and environmental data and how their back-end geoprocessing services were built with Python.

The Eastern Interconnection States' Planning Council (EISPC) Energy Zones Mapping Tool (EZMT) [Ezmt] was developed primarily to facilitate identification of potential energy zones or areas of high resource concentration for nine different low- or nocarbon energy resources, spanning more than 30 grid-scale energy generation technologies. The geographic scope is the Eastern Interconnection (EI), the electrical grid that serves the eastern

* Corresponding author: jkuiper@anl.gov

¥ Argonne National Laboratory

Copyright $(02014$ James A. Kuiper et al. This is an open-access article distributed under the terms of the Creative Commons Attribution License, which permits unrestricted use, distribution, and reproduction in any medium, provided the original author and source are credited.
United States and parts of Canada. The EZMT includes more than 250 map layers, a flexible suitability modeling capability with more than 35 pre-configured models and 65 input modeling layers, and 19 reports that can be run for user-specified areas within the EI. More background about the project is available from [Arg13].

Solar Energy Environmental Mapper (Solar Mapper) [Sol] provides interactive mapping data on utility-scale solar energy resources and related siting factors in the six southwestern states studied in the Solar Energy Development Programmatic Environmental Impact Statement [DOI12]. The application was first launched in December 2010, and a version that has been reengineered with open-source components is scheduled for launch in June 2014. Solar Mapper supports identification and screeninglevel analyses of potential conflicts between development and environmental resources, and is designed primarily for use by regulating agencies, project planners, and public stakeholders. More details about Solar Mapper can be found in [Sol13].

The Ecological Risk Calculator (ERC) [Erc] estimates risk in individual watersheds in the western United States to federally listed threatened and endangered species, and their designated critical habitats from energy-related surface and groundwater withdrawals. The approach takes into account several biogeographical characteristics of watersheds including occupancy, distribution, and imperilment of species, and their sensitivity to impacts from water withdrawals, as well as geophysical characteristics of watersheds known to include designated critical habitats for species of concern. The ERC is intended to help project planners identify potential levels of conflicts related to listed species (and thus the associated regulatory requirements), and is intended to be used as a preliminary screening tool.

Each of these Web-based mapping applications includes both vector (geographic data stored using coordinates) and raster (geographic data stored as a matrix of equally sized cells) spatial data stored in a relational database. For each application, Python was used to add one or more custom geoprocessing, modeling, or reporting services. The following section provides background on the software environment used, followed by specific examples of code with a discussion about the unique details in each.

One of the distinctive elements of geographic data management and processing is the need for coordinate reference systems and coordinate transformations (projections), which are needed to represent areas on the earth's oblate spheroid shape as planar maps and to manage data in Cartesian coordinate systems. These references appear in the code examples as "3857," the European Petroleum Survey Group (EPSG) Spatial Reference ID (SRID) reference for WGS84 Web Mercator (Auxiliary Sphere) 
and "102003," the USA Contiguous Albers Equal Area Conic projection commonly used for multi-state and national maps of the United States. These standardized EPSG definitions are now maintained by the International Association of Oil \& Gas Producers (OGP) Surveying \& Positioning Committee [OGP].

The Web Mercator projection has poor properties for many elements of mapping and navigation [NGA] but is used for most current Web-based mapping applications because of the wide availability of high-quality base maps in the Web Mercator projection from providers such as Google Maps. In the Solar Mapper project, we compared area computations in the southwestern United States using Web Mercator against the Albers Equal Area projection and found very large discrepancies in the results (Table 1).

The distortion inherent in world-scale Mercator projections is easily seen by the horizontal expansion of features, which increases dramatically in the higher northern and southern latitudes. In each of our projects, we chose to store local geographic data in Web Mercator to match the base maps and increase performance. However, for geographic processing such as generating buffers and computing lengths and areas, we first convert coordinates to the Albers Equal Area projection to take advantage of the improved properties of that projection.

\section{SOFTWARE ENVIRONMENT}

Each of these systems was built with a multi-tier architecture composed of a Javascript/HTML (hypertext markup language) interface built on Bootstrap [Btsrp], OpenLayers [OpLyr], and ExtJS [Sen]; a Web application tier built on Ruby on Rails [RoR]; a mapping tier implemented with GeoServer [Gsrvr]; a persistence tier implemented with PostGIS [PGIS]; and an analysis tier built on Python, PyWPS [PyWPS], GRASS [GRASS], and the spatial analysis functionality of PostGIS. These systems are deployed on Ubuntu [Ub] virtual machines running in a private VMware [VM] cloud. The Python-orchestrated analysis tier is the focus of this paper.

Many of the examples show geospatial operations using PostGIS. PostGIS extends the functionality of PostgreSQL for raster and vector spatial data with a robust library of functions. We found it to be well documented, reliable, and the "footprint analysis" tools we describe in the examples run significantly faster than similar tools we had previously developed with a popular commerical GIS framework.

\section{EXAMPLES}

One of the primary capabilities of each of our Web applications was using an area selected or drawn by the user for analysis (a "footprint"); collecting vector and raster data inside, intersecting, or near the footprint; and compiling it in a report. The first example shows the steps followed through the whole process, including the user interface, and later examples concentrate on refinements of the Python-coded steps.

\section{Full Process for Footprint Analysis of Power Plant Locations Stored as Point Features}

This example is from the EZMT and illustrates part of its Power Plant report. The user draws an area of interest over the map (Figure 1) and specifies other report parameters (Figure 2). The "Launch Report" button submits a request to the Web application

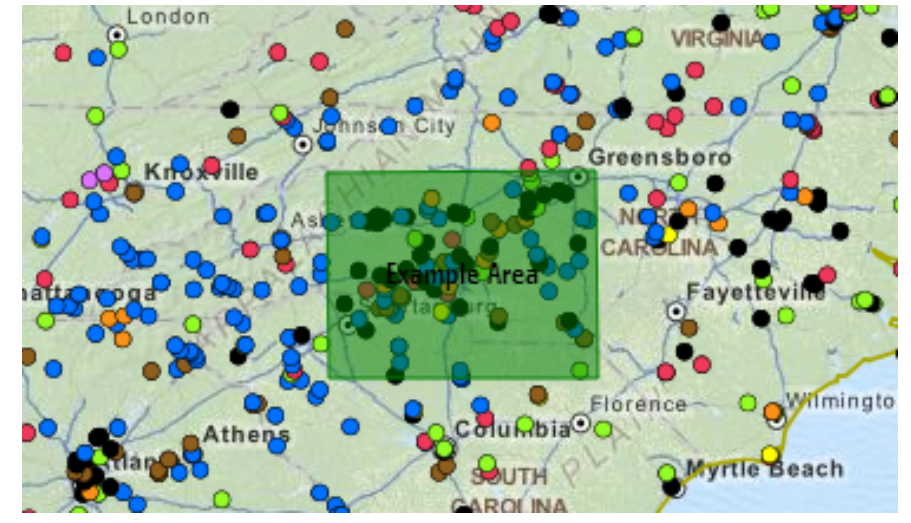

Fig. 1: EZMT Interface View of User-Specified Analysis Area and Power Plant Points

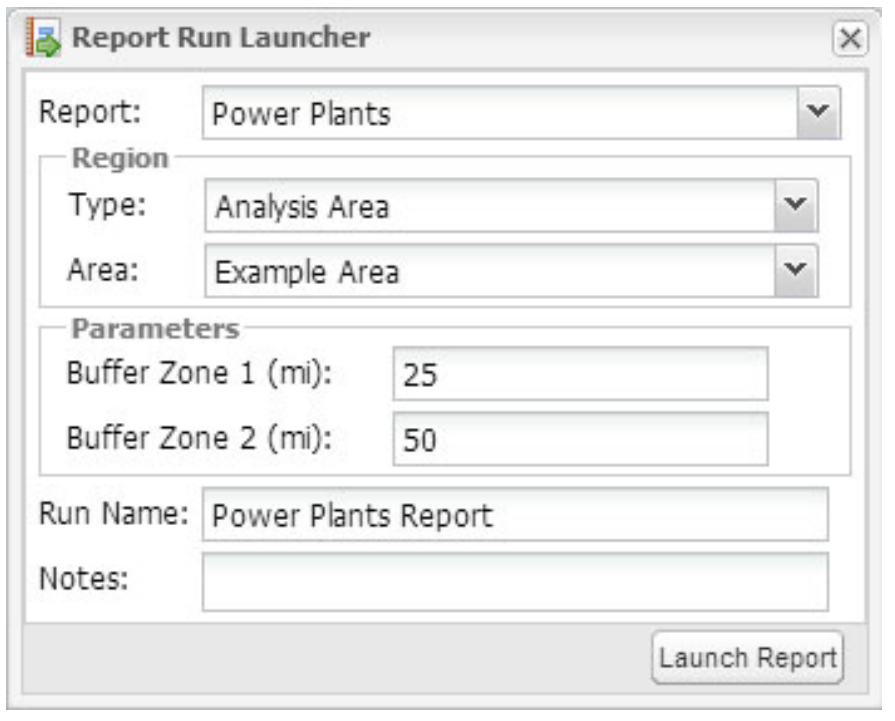

Fig. 2: EZMT Interface View of the Report Run Launcher

server to schedule, launch, track, and manage the report's execution.

The Web application initiates the report run by making a WPS request to the service, which is implemented in PyWPS. The request is an XML (extensible markup language) document describing the WPS "Execute" operation and is submitted via a hypertext transfer protocol (HTTP) POST. PyWPS receives this POST request, performs some basic validation and preprocessing, and routes the request to the custom WPSProcess implementation for that request. PyWPS then prepares the HTTP response and returns it to the application server. The code below illustrates the major steps used to generate the data for the report.

We use the psycopg 2 library to interact with the database, including leveraging the geographic information system (GIS) capabilities of PostGIS.

\section{\# Import PostgresSQL library for database queries import psycopg2}

The user-specified footprint corresponding to Figure 1 is hardcoded in this example with Web Mercator coordinates specified in meters and using the Well-Known Text (WKT) format.

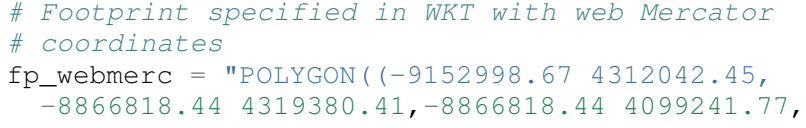




\begin{tabular}{|l|r|r|r|}
\hline \multirow{2}{*}{ Projection } & \multicolumn{3}{|c|}{ Area (square miles) } \\
\cline { 2 - 4 } & Large Horizontal Area & Large Vertical Area & Smaller Square Area \\
\hline Albers Equal Area & $7,498.7$ & $10,847.3$ & 35.8 \\
\hline Web Mercator & $13,410.0$ & $18,271.4$ & 63.0 \\
\hline Difference & $5,911.3$ & $7,424.1$ & 27.2 \\
\hline Percent Difference & $44 \%$ & $41 \%$ & $43 \%$ \\
\hline
\end{tabular}

TABLE 1: Comparison of Area Computations between the Web Mercator Projection and the Albers Equal Area Projection in the Southwestern United States

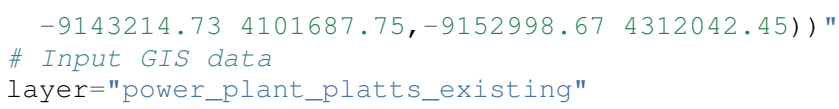

A database connection is then established, and a cursor is created.

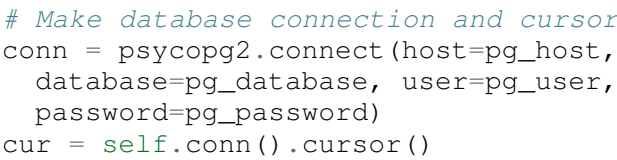

Structured Query Language (SQL) is used to (1) convert the Web Mercator footprint to the Albers Equal Area projection, (2) generate a buffer around the Albers version of the footprint, and (3) convert that buffer back to Web Mercator. In these sections, ST_GeomFromText converts WKT to binary geometry, and ST_AsText converts binary geometry back to WKT. Because WKT does not store projection information, it is given as a parameter in ST_GeomFromText.

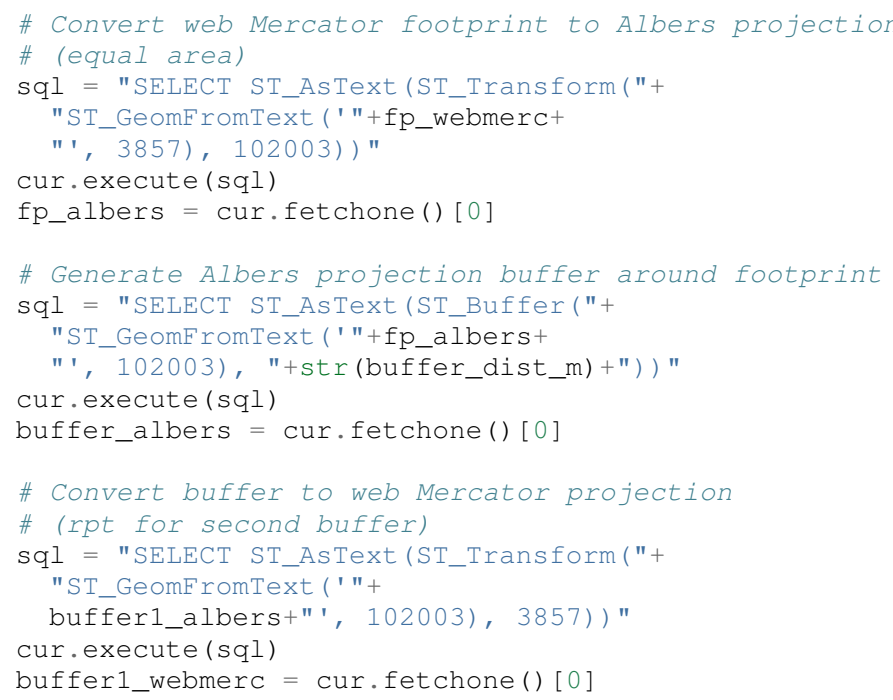

The previous steps are handled similarly for every report in an initialization method. The final SQL statement in this example retrieves data for the report content itself. The ST_Intersect S method queries the geometries in the power plant layer and returns the records intersecting (overlapping) the footprint. These records are summarized [count $(*)$, sum(opcap), and GROUP BY energy_resource] to provide content for the initial graph and table in the report. This SQL statement is repeated for the two buffer distances around the footprint.

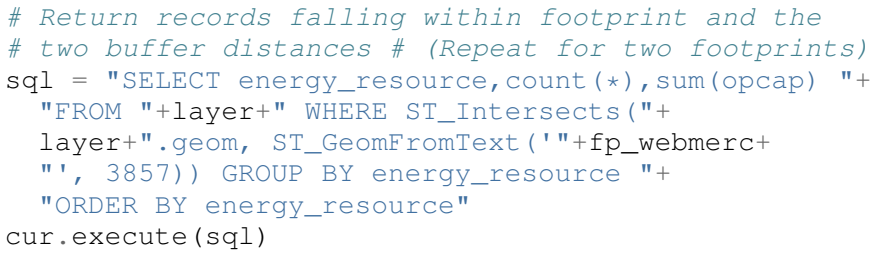

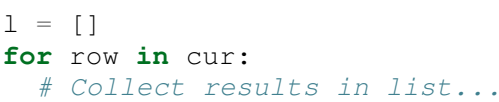

Once the data have been retrieved, the code compiles it into a Python dictionary which is rendered and returned as a JSON document (excerpt below). This document is retained by the application for eventual rendering into its final form, HTML with the graphs built with ExtJS. Figure 3 shows a portion of the report.

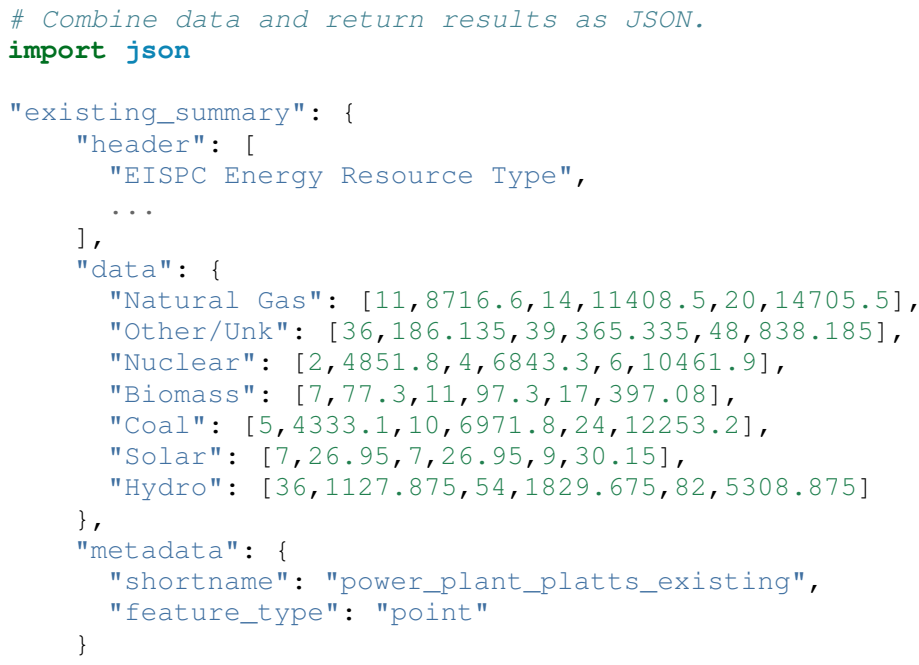

Footprint Analysis of Transmission Lines Stored as Line Features

Another EISPC report uses a user-specified footprint to analyze electrical transmission line information; however, rather than only listing features inside the footprint as in the previous example, (1) in contrast to points, line features can cross the footprint boundary; and (2) we want to report the total length of the portion within the footprint rather than only listing the matching records. Note that ST_Intersects is used to collect the lines overlapping the footprint, whereas ST_Intersection is used to calculate lengths of only the portion of the lines within the footprint. In addition, the coordinates are transformed into the Albers Equal Area projection for the length computation.

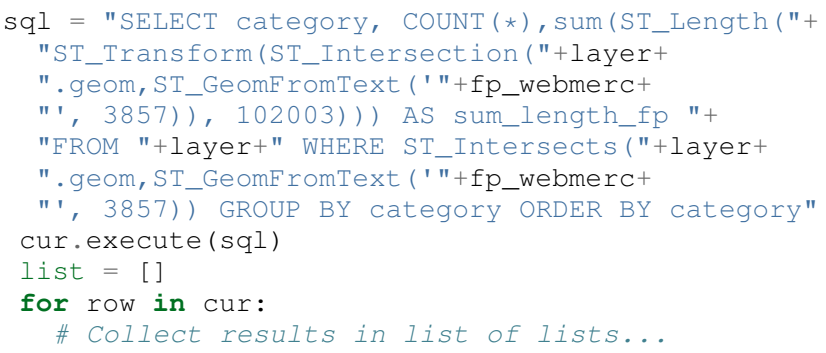




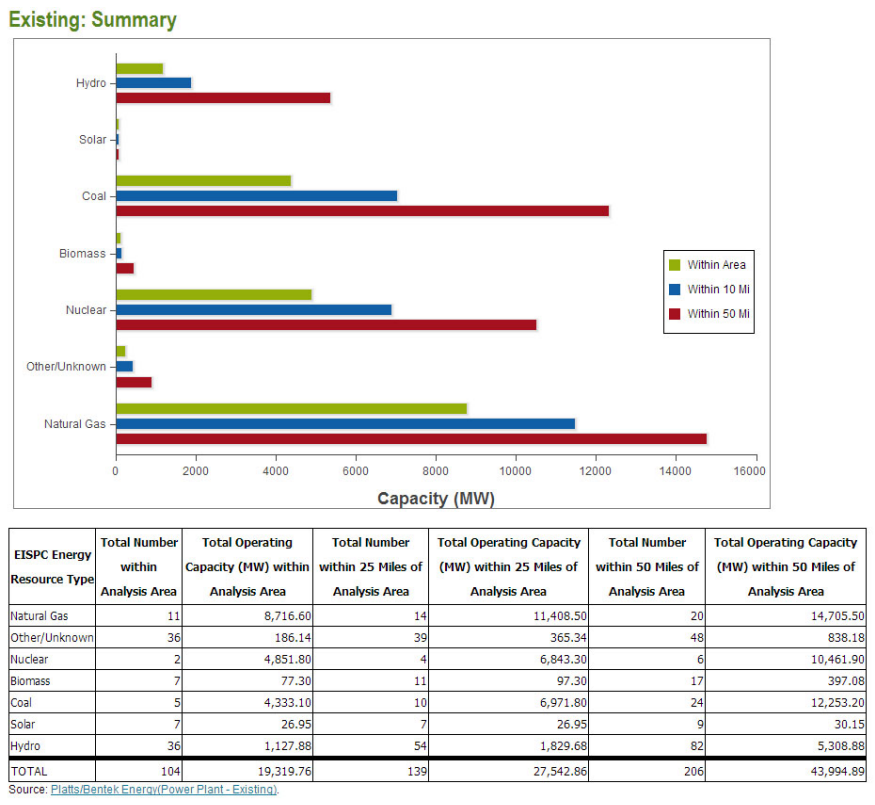

Fig. 3: Portion of EZMT Power Plant Report

Results in JSON format:

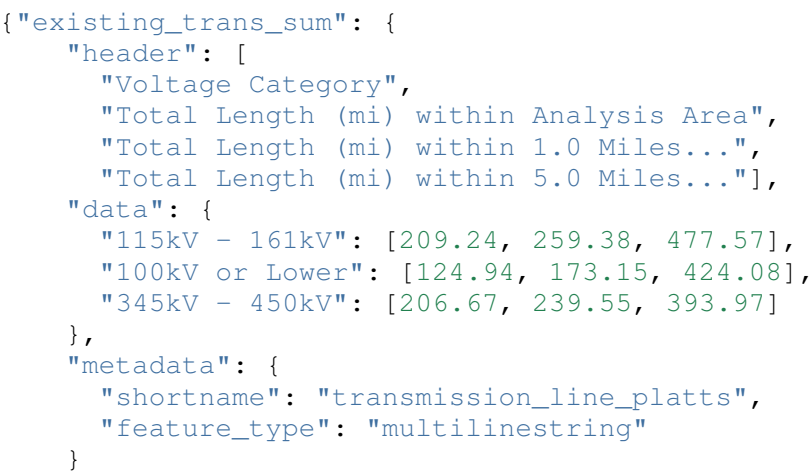

Footprint Analysis of Land Jurisdictions Stored as Polygon Features

In the Solar Mapper report for Protected Lands, the first section describes the land jurisdictions within a footprint, and a 5mile area around it, with areas. The sma_code field contains jurisdiction types. The query below uses ST_Intersects to isolate the features overlapping the outer buffer and computes the areas within the buffer and footprint for each jurisdiction that it finds for a particular report run. For the area computations, ST_Intersection is used to remove extents outside of the footprint or buffer, and ST_Transform is used to convert the coordinates to an Albers Equal Area projection before the area computation is performed.

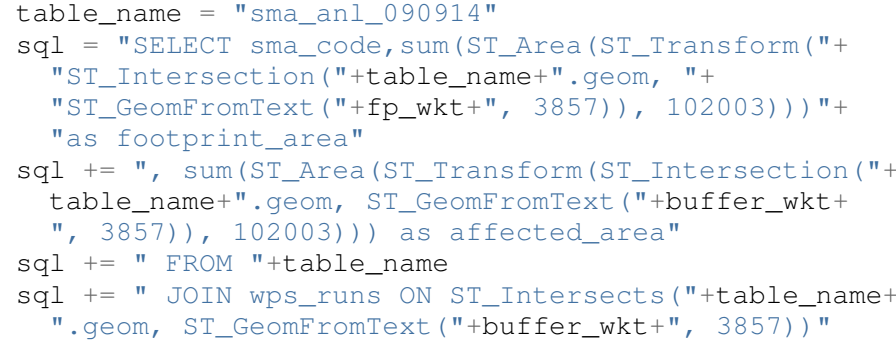

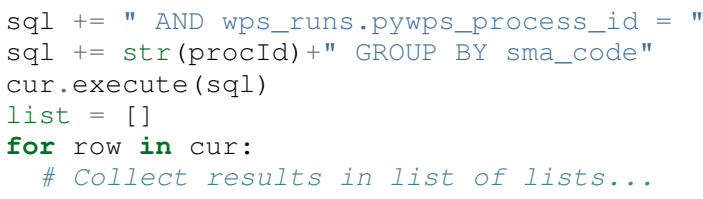

\section{Footprint Analysis of Watershed Areas Stored as Polygon Fea- tures, with Joined Tables}

The Environmental Risk Calculator [?] involves analysis of animal and plant species that have been formally designated by the United States as threatened or endangered. The ERC estimates the risk of water-related impacts related to power generation. Reports and maps focus on watershed areas and use U.S. Geological Survey watershed boundary GIS data (stored in the huc_8 table in the database). Each watershed has a Hydrologic Unit Code (HUC) as a unique identifier. The huc8_species_natser table identifies species occurring in each HUC, and the sensitivity table has further information about each species. The ERC report uses a footprint analysis similar to those employed in the previous examples. The query below joins the wps_runs, huc8_poly, huc8_species_natser, and sensitivity tables to list sensitivity information for each species for a particular report run for each species occurring in the HUCs overlapped by the footprint. Some example results are listed in Table 2.

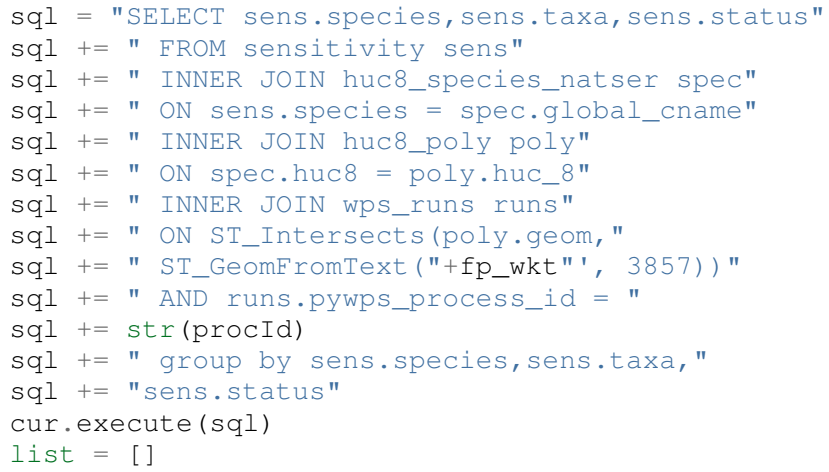




\begin{tabular}{lll}
\hline Species & Taxa & Status \\
California Red-legged Frog & Amphibian & T \\
California Tiger Salamander - Sonoma & Amphibian & E \\
County & & \\
Colusa Grass & Plant & T \\
Conservancy Fairy Shrimp & Invertebrate & E \\
Fleshy Owls clover & Plant & T \\
\hline
\end{tabular}

TABLE 2: Example Ecorisk Calculator Results Listing Threatened and Endangered Species Occurring in a Watershed

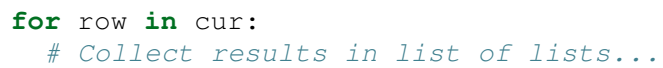

\section{Footprint Analysis of Imperiled Species Sensitivity Stored as Raster (Cell-based) Data}

Many of the layers used in the mapping tools are stored as raster (cell-based) data rather than vector (coordinate-based) data. The $\mathrm{ST}$ _Clip method can retrieve raster or vector data and returns the data within the footprint. The WHERE clause is important for performance because images in the database are usually stored as many records, each with a tile. ST_Intersects restricts the much more processing-intensive ST_Clip method to the tiles overlapping the footprint. When the footprint overlaps multiple image tiles, multiple records are returned to the cursor, and results are combined in the loop.

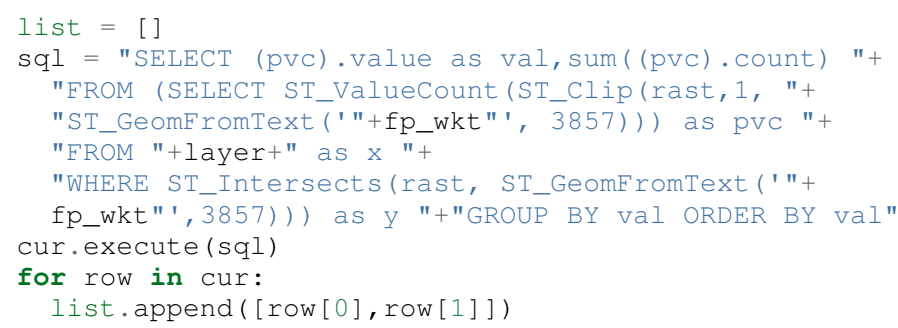

Results in JSON format:

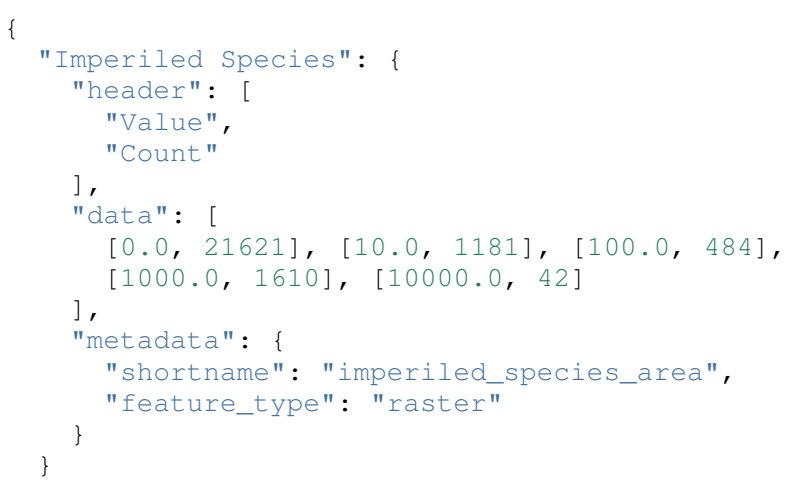

\section{Elevation Profile along User-Specified Corridor Centerline Us- ing Elevation Data Stored as Raster Data}

The Corridor Report in the EZMT includes elevation profiles along the user-input corridor centerline. In this example, an elevation layer is sampled along a regular interval along the centerline. First, the coordinate of the sample point is generated with ST_Line_Interpolate_Point, next, the elevation data are retrieved from the layer with $\mathrm{ST}_{\text {_Value. }}$

$\mathrm{d}=\{\}$

$\mathrm{d}[$ 'data'] $=[]$

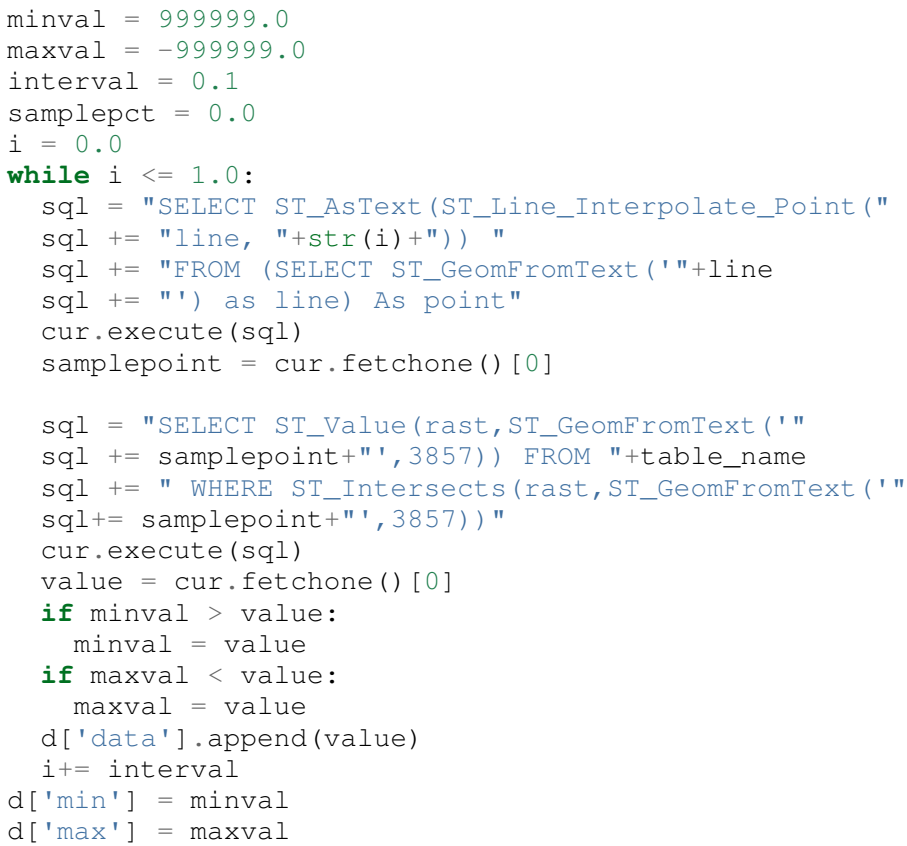

Results:

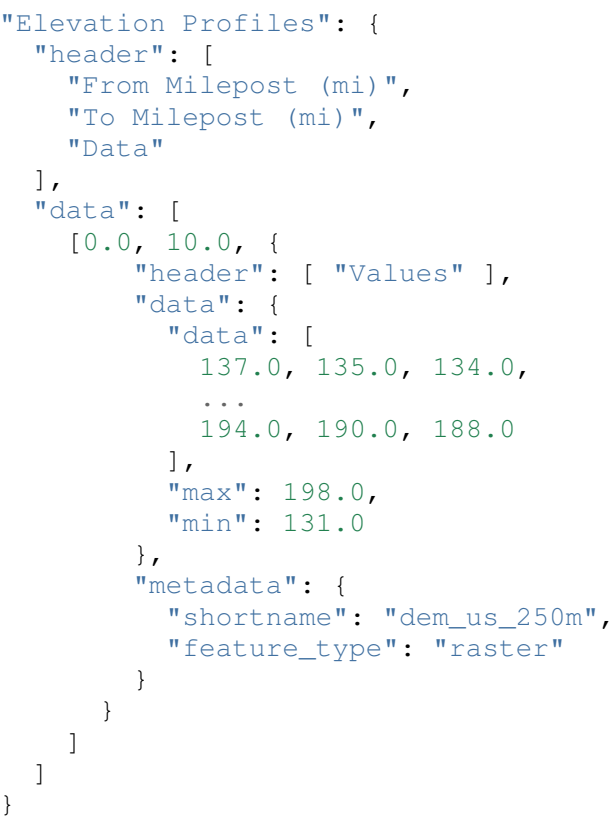

\section{Footprint Analysis of Raster Population Density Data}

In this example, the input data consist of population density values in raster format, and we want to estimate the total population within the footprint. As in the previous example, ST_Intersects is used in the WHERE clause to limit the tiles processed by the rest of the query, and multiple records will be output if the footprint overlaps multiple tiles. First, image cells overlapped by the footprint are collected and converted to polygons (ST_DumpAsPolygons). Next, the polygons are trimmed with the footprint (ST_Intersection) to remove portions of cells outside the footprint and are converted to an equal area projection (ST_Transform); and then the area is computed. Finally, the total population is computed (density * area), prorated by the proportion of the cell within the footprint.

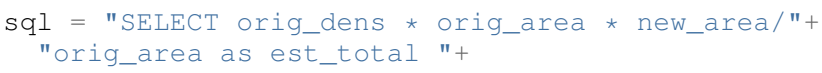




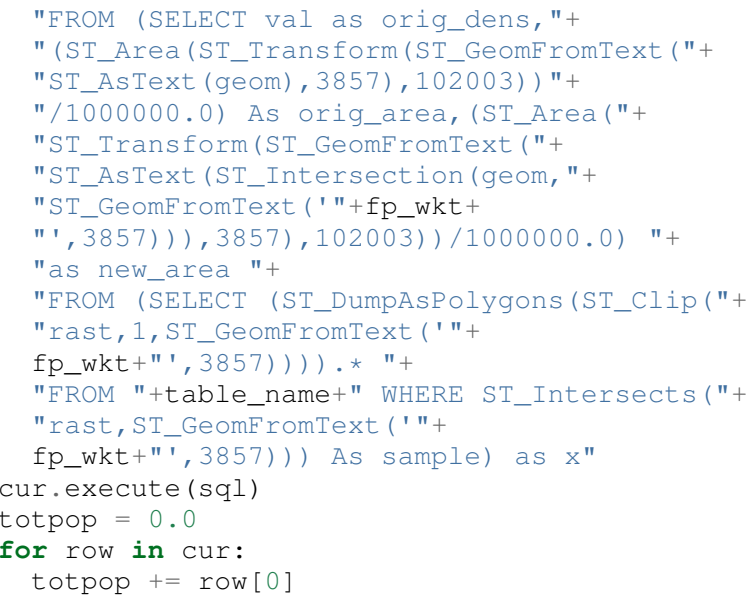

\section{Computation of Suitability for Wind Turbines Using Raster Data Using GRASS}

The suitability models implemented in the EZMT use GRASS software for computations, which are accessed in Python through WPSs. The code below shows the main steps followed when running a suitability model in the EZMT. The models use a set of raster layers as inputs, each representing a siting factor such as wind energy level, land cover, environmental sensitivity, proximity to existing transmission infrastructure, etc. Each input layer is coded with values ranging from 0 (Completely unsuitable) to 100 (Completely suitable), and weights are assigned to each layer representing its relative importance. A composite suitability map is computed using a weighted geometric mean. Figure 4 shows the EZMT model launcher with the default settings for land-based wind turbines with 80-meter hub heights.

Processing in the Python code follows the same steps that would be used in the command-line interface. First, the processing resolution is set using $g . r e g i o n$. Then, the input layers are processed to normalize the weights to sum to 1.0 (this approach simplifies the model computation). Next, an expression is generated, specifying the formula for the model, and $r$.mapcalc is called to perform the model computation. r.out.gdal is used to export the model result from GRASS format to GeoTiff for compatibility with GeoServer, and the projection is set using gdal_translate from the Geospatial Data Abstraction Library [GDAL] plugin for GRASS.

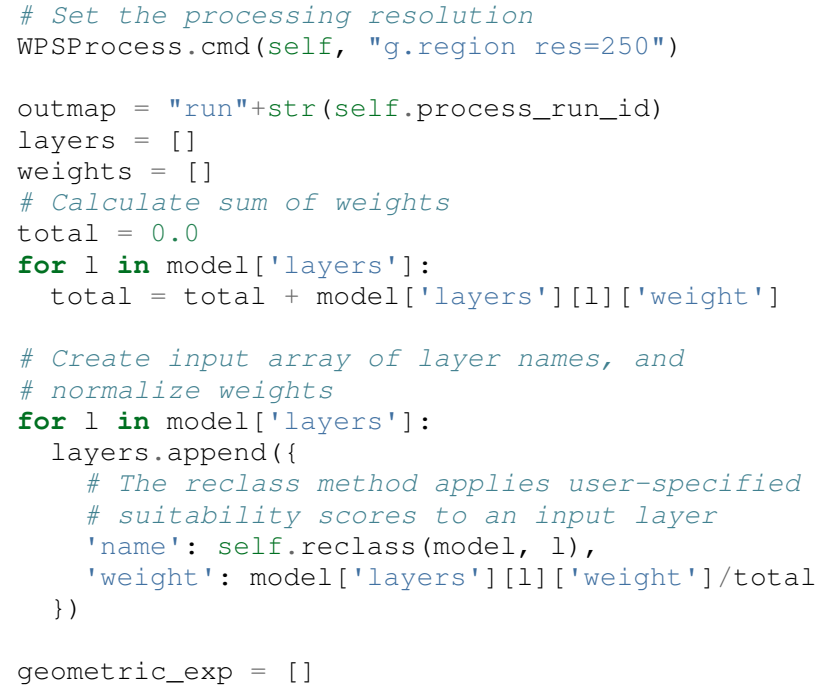

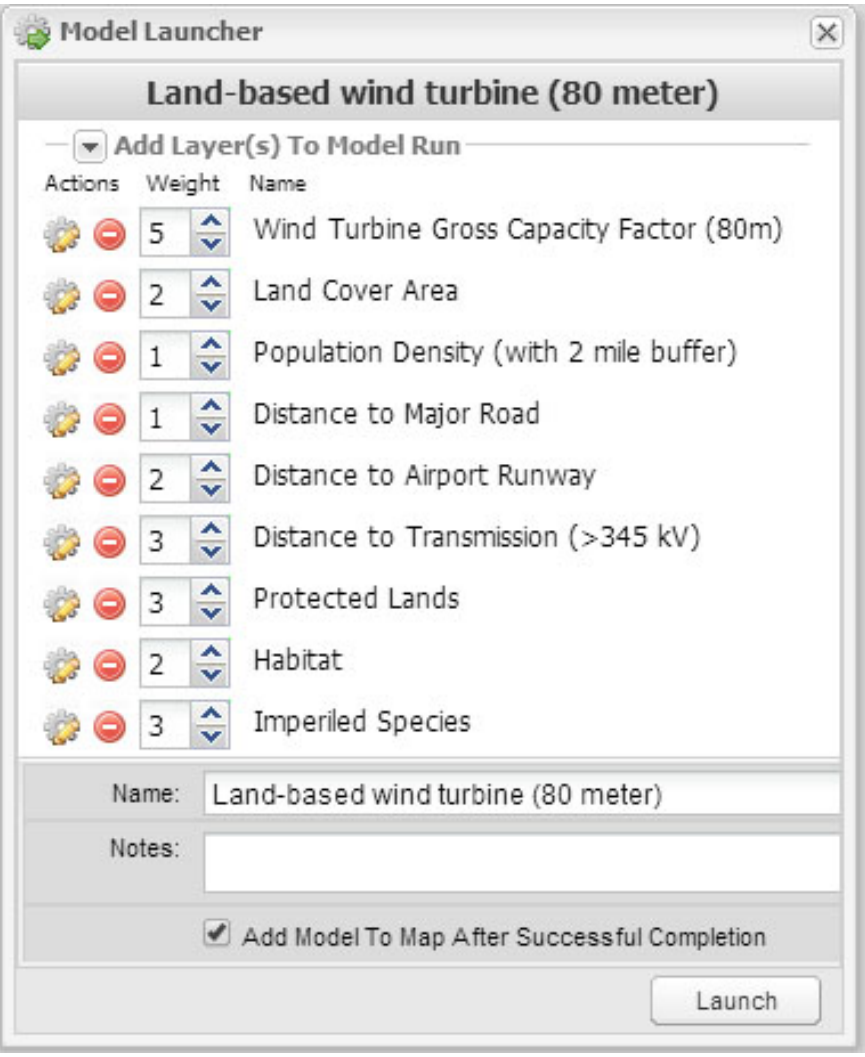

Fig. 4: Land-based Wind Turbine Suitability Model Launcher in the EISPC Energy Zones Mapping Tool

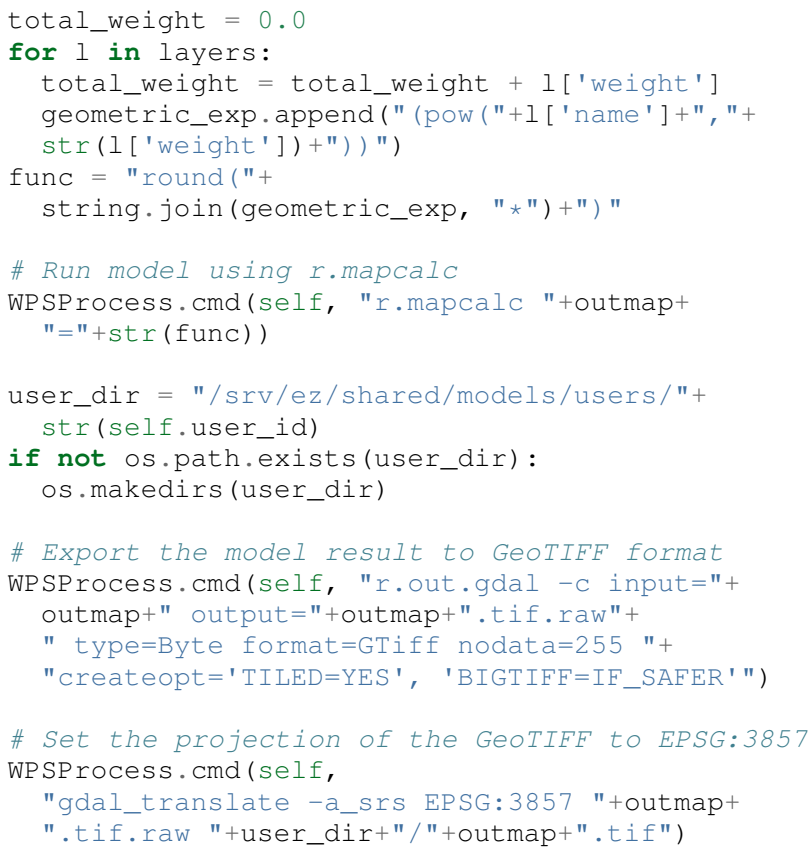

\section{CONCLUSIONS}

Python is the de-facto standard scripting language in both the open source and proprietary GIS world. Most, if not all, of the major GIS software systems provide Python libraries for system integration, analysis, and automation, including ArcGIS, GeoPandas [GeoP], geoDjango [geoD], GeoServer, GRASS, PostGIS, pySAL [pySAL], and Shapely [Shp]. Some of these systems, such 
as ArcGIS and geoDJango, provide frameworks for web-based mapping applications different from the approach we described in the SOFTWARE ENVIRONMENT section. While it is outside the scope of this paper to discuss the merits of these other approaches, we recommend considering them as alternatives when planning projects.

The examples in this paper include vector and raster data, as well as code for converting projections, creating buffers, retrieving features within a specified area, computing areas and lengths, computing a raster-based model, and exporting raster results in GeoTIFF format. All examples are written in Python and run within the OGC-compliant WPS framework provided by PyWPS.

One of the key points we make is that the Web Mercator projection should not be used for generating buffers or computing lengths or areas because of the distortion inherent in the projection. The examples illustrate how these computations can be performed easily in PostGIS. We chose to use the Albers Equal Area projection, which is commonly used for regional and national maps for the United States. Different projections should be used for more localized areas.

So far our Web-based mapping applications include fairly straightforward analysis and modeling services. However, the same approaches can be used for much more sophisticated applications that tap more deeply into PostGIS and GRASS, or the abundant libraries available in the Python ecosystem. Matplotlib, NetworkX, NumPi, RPy2, and SciPy can each be integrated with Python to provide powerful visualization, networking, mathematics, statistical, scientific, and engineering capabilities.

\section{ACKNOWLEDGMENTS}

This work was supported by the U.S. Department of Energy, Office of Electricity Delivery and Energy Reliability; and the U.S. Department of Interior, Bureau of Land Management, through U.S. Department of Energy contract DE-AC02-06CH11357. The submitted manuscript has been created by the University of Chicago as Operator of Argonne National Laboratory ("Argonne") under contract No. DE-AC02-06CH11357 with the U.S. Department of Energy. The U.S. Government retains for itself, and others acting on its behalf, a paid-up, nonexclusive, irrevocable worldwide license in said article to reproduce, prepare derivative works, distribute copies to the public, and perform publicly and display publicly, by or on behalf of the Government.

\section{RefERENCES}

[Arg13] Argonne National Laboratory, Energy Zones Study: A Comprehensive Web-Based Mapping Tool to Identify and Analyze Clean Energy Zones in the Eastern Interconnection, ANL/DIS-13/09, September 2013. Available at https://eispctools.anl.gov/document/21/file

[Btsrp] http://getbootstrap.com

[DOI12] U.S. Department of the Interior, Bureau of Land Management, and U.S. Department of Energy, Final Programmatic Environmental Impact Statement for Solar Energy Development in Six Southwestern States, FES 12-24, DOE/EIS-0403, July 2012. Available at http://solareis.anl.gov/documents/fpeis

[Erc] http://bogi.evs.anl.gov/erc/portal

[Ezmt] http://eispctools.anl.gov

[GDAL] http://www.gdal.org

[geoD] http://geodjango.org

[GeoP] http://geopandas.org

[Ggl] http://maps.google.com

[GRASS] http://grass.osgeo.org

[Gsrvr] http://geoserver.org

[NGA] http://earth-info.nga.mil/GandG/wgs84/web_mercator/index.html

[OGP] http://www.epsg.org
[OpLyr] http://openlayers.org

[PGIS] http://postgis.net/docs/manual-2.0/reference.html

[pySAL] http://pysal.readthedocs.org/en/v1.7

[PyWPS] http://pywps.wald.intevation.org

[RoR] http://rubyonrails.org

[Sen] http://www.sencha.com/products/extjs

[Shp] http://pypi.python.org/pypi/Shapely

[Sol] http://solarmapper.anl.gov

[Sol13] Kuiper, J., Ames, D., Koehler, D., Lee, R., and Quinby, T., "WebBased Mapping Applications for Solar Energy Project Planning," in Proceedings of the American Solar Energy Society, Solar 2013 Conference. Available at http://proceedings.ases.org/wp-content/uploads/ 2014/02/SOLAR2013_0035_final-paper.pdf.

[Ub] http://www.ubuntu.com

[VM] http://www.vmware.com 